\title{
Proposal to Improve Mobility in Service Oriented Architecture
}

\author{
Dalal Aljebry ${ }^{a}$, Rizwan Qureshi ${ }^{b, *}$ \\ ${ }^{\mathrm{a}, \mathrm{b}}$ Faculty of Computing and Information Technology, King Abdulaziz University, Jeddah, Saudi Arabia
}

\begin{abstract}
Service Oriented Architecture (SOA) is a standard to implement and design applications. It is a channel of communication between service consumer (SC) and service provider (SP). SP offers a service and SC uses a service. Mobile web service verifier component, of SOA architecture, matches best service that is called. Mobile web service verifier component finds a best match of particular service in SP if it is available by the SP. Therefore, there is a pressing need to develop a component that can create a service if it is not found by mobile web service verifier component. This problem is addressed by proposing a new component for SOA architecture called service upgrade (SU) to increase and improve the service creation speed and satisfy SC and SP needs. The questionnaire is used as a research design to evaluate the proposed solution by providing qualitative data. The results show that the majority of respondents are in favor of the proposed solution. It is anticipated that the proposed solution will cater the industrial problem in hand.
\end{abstract}

Index Terms: Service Oriented Architecture, Mobile Services, Service Provider, Service Consumer and Mobile Web Service Verifier.

(C) 2017 Published by MECS Publisher. Selection and/or peer review under responsibility of the Research Association of Modern Education and Computer Science.

\section{Introduction}

With the increase of using mobile devices in our daily tasks from smallest function such as making call to many complex applications such as email, location services and banking, etc. Mobile technology allows users to carry large numbers of resources and to access them wherever they find it convenient by using portable devices such as PCs, smartphones and hand-held [3]. Service oriented architecture (SOA) is a popular and effective software engineering approach. It helps the software development companies to reuse previously developed components. SOA is a software engineering technique that is used to build loosely coupled distributed systems [3]. SOA consists of three main components that are service consumer (SC), service provider (SP) and Service Registry (SR). Each component of SOA architecture has to perform a specific task to develop distributed systems. Fig. 1 illustrates the layout of SOA components. A mobile service is created by SP. SP distributes service interface and access information to SR. SC invokes to SR for a mobile service. In

* Corresponding author:

E-mail address: rmuhammd@kau.edu.sa 
addition to find and bind mechanism, mobile client service requests one of services from SP and locates an entry in SR. SC sends a service request to SP in order to execute function of service. SR is a services reference database and it contains information of services including service interfaces, definition, and parameters. The data and metadata are stored in a repository.

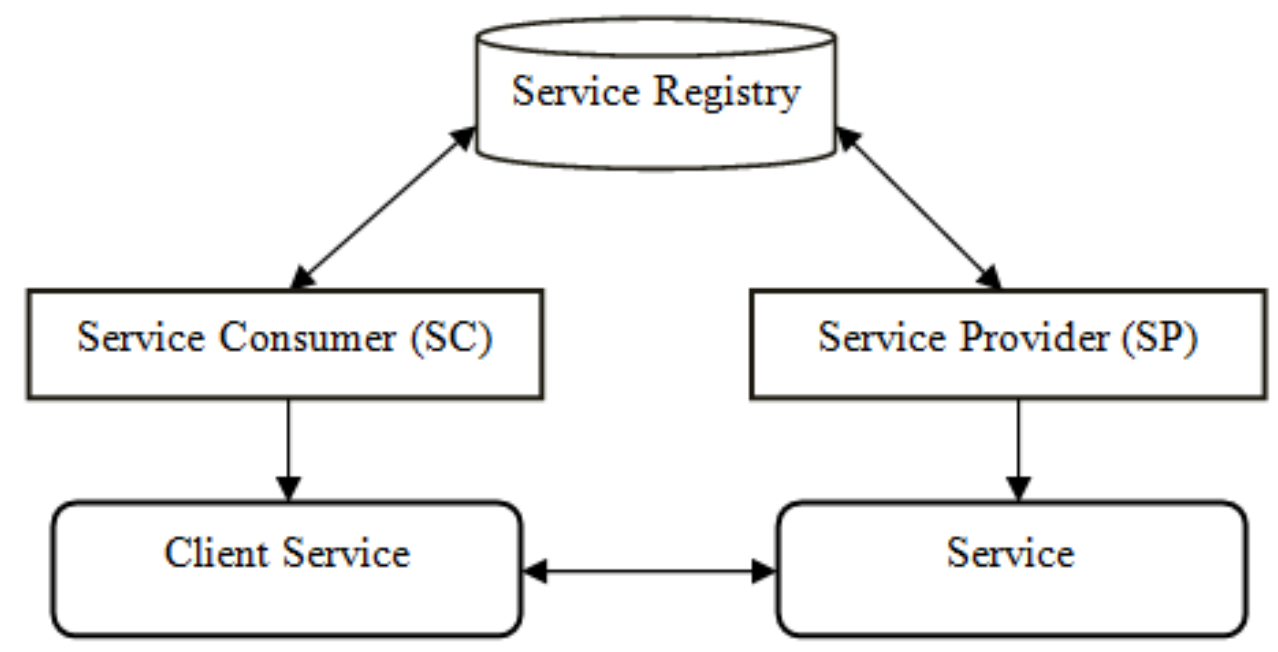

Fig.1. Service Oriented Architecture.

SP requests to context Interpreter for context data. Rule checker receives SP request from context interpreter and it checks for valid context. Context interpreter makes the data available to SP after collecting from context database. Context database has an inference rule part that contains set of facts and rules. Inference rule set defines rules for each context. Rule checker checks the rule to match between particular context and predefined rules using inference rule set. Context classifier gets data from context database. Context transformer applies rules on the receiving data. Context database creates context data records that are requested by Context Interpreter. Then it does couple task; sends to Context Interpreter a context data and updated SR context information. To store context in Context Database, it must classify it into different categories by Context Classifier. Context data aggregates using context data aggregator that is received from local and external database. The benefit of this aggregation is to catch information details of specific groups based on context parameters. There is a limitation of provide data from a local database that minimize generalized data requirement and placed on the mobile device. Database contains additional detailed of context information because it is from a variety of database and devices such as mobile device [13].

Mobile Web Service (MWS) is a component of SOA architecture [6]. It is a third party web service to verify services between SP and SC. Quality of Web Service (QoWS) verifies required resources for each operation of the service. QoWS ensures SP valid conformance and assist MWS to find the best matching service [6].

Mobile applications have limited functionalities such as restricted mobile hardware resources, issue of disconnectivity, battery life and computation power. For instance, iPhone 6 released on 19 September, 2014, 1810 mAh battery, Apple A8 system-on-chip, an M8 motion co-processor, and 4.7-inch screen. Laptops features are high-speed multiprocessors, RAM measured in gigabytes, and 13.3-inch screen. In line with these differences, communications between a web Service and a mobile client could lead to limited resources mobile applications and devices. Indeed, a mobile application has to present mobility-aware functionalities to ensure that mobile client is served by the desired web service [6].

SOA has the mobility feature because of the following: 
One of the characteristics of SOA is that it's defined as a public interface so that the provider and requester interact through a heterogeneous environment.

- SOA is the best choice for solving problems of mobile devices.

- Web services support the mobile application.

- SOA supports modeling in mobile ambient by extending it with many conceptualizations.

- Ubiquitous systems designed by using SOA; it offers services anywhere and anytime requested.

- Others report that SOA is appropriate for mobile services.

This paper is written to propose a new component naming service upgrade (SU) as an addition into SOA architecture. SU will create a service if the desired matching service is not available.

The paper is organized as follows. Section 2 outlines the related work. Section 3 describes the problem statement. Section 4 depicts the details of the proposed solution. Section 5 provides the validation of proposed solution.

\section{Related Work}

The reviews of mobility in Service Oriented Architecture (SOA) could be categories into three directions: mobile services, mobile ambient and web services. The SOA features are for multi-device user access to services and non-robust connections environments. It makes mobile application composition based on SOA implementations natural components.

Shrestha [1] proposed advantages of implementing SOA and introduce Context-Aware Mobile Application (CAMA). Combined SOA and CAMA provided a limitations solution of conventional frameworks [1]. Romsaiyud and Pemchaiswadi [2] implemented SOA and CAMA. It will automatically help a client to configure certain aspects of its service by an optional SR. When SP executes the changes according to service details of the registry then notified SC of changes and configured its service client in order to communicate service [2].

A connectivity network is sharing a common medium for communicating between providers and users. User or customer may connect through a mobile cellular network that do not use IP connectivity, optical network, or varies wires area network. The SP should not concern with the underlying network. Hoang and Pham [3] formed a Service Oriented Network Architecture (SONA) to simplify services and applications development. SONA conceived consideration of the necessary requirements of generic Internet applications from the perspective of service and network providers. The objective is to address SOA application deployment pattern, IP-converged networks structure, user location, and mobility of device [2].

The focus is on the composed services available time and each service is expressed by its name and mobile prediction information [4]. A heuristic algorithm is developed and simulated to test each service [4]. In urban areas using Integrated Real-time Mobility Assistant (IRMA), Mobile platform is based on Service Oriented Architecture/Event Driven Architecture (SOA/EDA) technology to access open data using General Transit Feed Specification (GTFS) format [5]. IRMA software system focuses on future scenario of personal mobility. It is based on green, shared and public transports [5]. Choudhury et al. [6] addressed mobility, link failure, and delay of dynamic service composition framework in SOA according to Quality of Service (QoS). Choudhury et al. focus on a single point of link failure [6].

Abolfazli et al. [7] simulate new architecture naming Market Oriented Mobile Cloud Computing (MOMCC). MOMCC framework is used to develop mobile cloud applications. It stimulates mobiles publishing, discovering, and hosting Services. MOMCC decreases latency of long wide area network and it also creates a chance for business for a purpose of encourages Service hosting to be used by mobile users. Ubiquitous systems are illustrated with respect to users' services without paying attention to the users' locations. The main limitations of mobile systems are scalability, connectivity, adaptability, liability, and ease-of-use that are needed in a typical ubiquitous system. Based on Service Governance principles in SOA framework, a 
ubiquitous wireless mobile system is designed implemented and experimented naming uSG- Health [7].

Table 1. The Limitations of Related Work.

\begin{tabular}{ll}
\hline Reference & Limitation \\
\hline$[1]$ & CAMA processes requests only if context database contains the context data and SR has the metadata. \\
{$[2]$} & $\begin{array}{l}\text { Need permanently and continuously updated with an enormous database however SP must create many services } \\
\text { in order to satisfy the consumer. }\end{array}$ \\
{$[3]$} & Security and validations are the main concerns of study \\
{$[4]$} & Developed only algorithms. Focused their research on a static case not on a dynamic model. \\
{$[5]$} & IRMA is in primary implementation stage with small lab demos only.
\end{tabular}

[6] Face a single point of link failure. Forwards a framework without a concrete implementation and optimize the quality of an omnipresent service according to QoS only of each service in isolation without considering how these services are composed together.

MOMCC architecture is just simulation case study that not be implemented yet.

[8] It well have enormous EHR database. It needs to update Constantly

[9] It has the flexibility but with the cost since such dynamic reconfigurations had a significant impact on application execution. Focus on low dynamic environments.

[10] There is a need to test the platform in more scenarios to generalize the results.

[12] Not a complete model-driven architecture

[13] No test cases for trust and use real application of Quality of Web Service (QoWS) which relying on MWS. No action when Mobile Web Service Verifier did not find a best match of particular service in SP.

[14] Focus in SOA only. Don't provide ways of generating documentation of the software architecture. Software platform built in old and no longer commercially available (not for mobile applications). Providing a platform with toolsets for a mobile device but there are some programs that cannot emerge some of the mobile technologies.

[15] The QoS model focus on the representation of knowledge rather than a language in order to describe QoS.

[16] User privacy and integrity are not considered.

[17] The proposal needs to be tested in a practical environment to check its effectiveness and applicability.

[18] The limitations of MCC are not considered. It is also not covered that how to establish set up in an educational environment to take the full benefits of MCC.

[19] The validation of the proposal is not provided. The proposed architecture to set up MCC environment is also not provided.

[20] The proposed mobile cloud computing educational tool can run only on Android platform. There is a need to enhance its working on other mobile platforms. The proposed tool is not validated using a case study to conclude and generalize the results.

[21] The proposed architecture needs to be implemented to check its effectiveness.

[22] The proposed research is an idea and the proof of concept is required. 
SOA is combined with applying service governance. An open source tool (JMeter) is used to test the performance of a system [8]. A framework is proposed to manage dynamic service-oriented component architecture [9]. The objective is to analyze the influence of dynamic reconfigurations. Secondly, missing dependencies are resolved of running applications. Both component based software development and service oriented computing offer building blocks [9]. A novel mobile social networking platform (MS2A) is proposed to ease application developments using SOA [10]. The service and application are two layers of MS2A platform.

Liu and Cheung [11] discuss characteristics and challenges of energy and performance bugs using Android applications. A case study is conducted to conclude the results [11]. Ali et al. [12] describe a tool for mobile ambient supporting Ambient-SoaML SOA. The current version of tool provides structural characteristics and it does not cover dynamic behavior [12]. Serhani and Benharref [13] propose an architecture naming Mobilityaware web services. The architecture is based on quality of web services and resources requirements. Sherriff [14] proposes a framework to develop mobile applications to teach SOA. Mabrouk et al. [15] present quality of service (QoS) model to improve the web service quality model (WSQM).

Cloud computing term is coined in 2007 [16]. It is an active area of research due to dynamic infrastructure, quality of services (QoS), guaranteed computing environments and configurable software services. There are several definitions of cloud computing but most of researchers are generally agreed on a number of key aspects $[16,17]$. M-learning using cloud is a new and leading edge research area and it is known as mobile cloud computing (MCC). MCC uses cloud infrastructure to develop business apps for mobile devices. The data processing and storage are done over cloud and results are displayed on the screen or communicated via speakers. The mobile network operators will be benefited through MCC like less cost and dependency on specific platform. Following are the main advantages of MCC [18].

- Economical (It does not requiring expensive hardware and software).

- Scalability.

- Robustness.

- Ubiquitous.

- Improved battery time of mobile devices.

- More computation power.

- Backup.

- Security.

Hardware/software constraints of mobile learning are discussed [19]. Cloud computing based m-learning system model, function and architecture are illustrated. The proposal needs to be validated using quantitative and qualitative techniques [19]. A tool is introduced to take benefits of cloud computing and mobile learning [20]. It is helpful to handle pictures, videos and complicated calculations. The tool is bundled with many algorithms such as de-blurring, de-noising and faces detection. The tool runs only on Android platform and it needs to be tested using controlled or uncontrolled case study to judge its effectiveness and generalize the results. Two proposals are presented to improve the present educational system by integrating cloud computing and mobile learning [21, 22]. The both proposals are discussed without providing proof of concept, architecture and practical implementation details. The limitations of reviewed paper are shown in Table 1. 


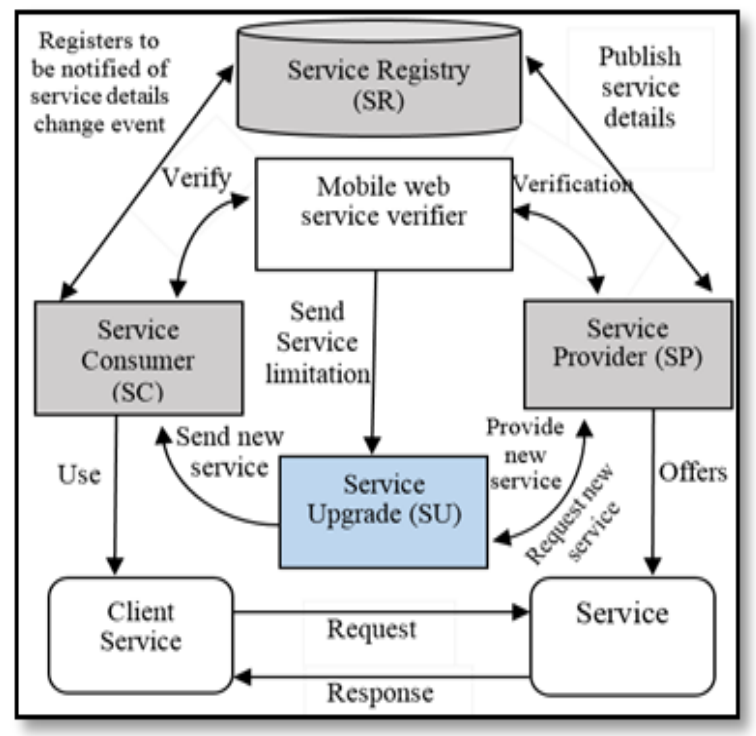

Fig.2. Mobility in Service Oriented Architecture (Request and Response).

\section{Problem Statement}

SOA is a software engineering technique to build loosely coupled distributed systems. It's a way to communicate between SC and SP components. Mobile web service verifier is a component in SOA architecture to verify the best matching service [13]. There are high chances that Mobile web service verifier component does not find the desired service between SC and SP. For that reason, there is a need of a new component to upgrade the mobility of SOA to serve the purpose $[2,13]$.

\section{The Proposed Solution}

There are several enhancement of SOA $[2,7,13]$ to add new features/components. The paper is written to propose a novel component named SU to enhance the services of SP by increasing mobility of SOA. Fig. 2 shows the proposed integration of SU in SOA. There are many benefits of SU component. SU is a new component of SOA architecture to enhance its functionality. SU acquires to SP for the desired service and deliver new services to SC when mobile web service verifier does not find the best matching service. It sends service limitation (consumer needs) to SU component. SU searches to find the best match from the set of newly untested services. SU will find the best matching service to deliver or it will combine multiple services to complete the service that is acquired by the consumer.

Table 2 illustrates the main components of SOA. Following are the benefits of including SU component in SOA.

- Give Mobile Web Service Verifier component action procedures in a situation of limited service in SP.

- Increase and improve the service creation speed

- Rapid discover of service limitation.

- Serve consumer requirements and needs.

- Find the error and restriction of services

- Test the newly created service. 
Table 2. The Main Components of SOA.

\begin{tabular}{|c|c|}
\hline Main Components of SOA & Functionalities \\
\hline \multirow[b]{4}{*}{ Mobile web service verifier } & $\begin{array}{l}\text { - } \quad \text { Produce mobile service } \\
\text { - } \quad \text { Distributes service interface and access information to the SR }\end{array}$ \\
\hline & Services reference database \\
\hline & Request for service \\
\hline & $\begin{array}{l}\text { - } \quad \text { Provides services verification to MWS consumers and providers. } \\
\text { Send a consumer needs of service to SU in a case of not finding the best match } \\
\text { service in SP }\end{array}$ \\
\hline SU & $\begin{array}{l}\text { - } \\
\text { - } \\
\text { - } \\
\text { - }\end{array}$ \\
\hline
\end{tabular}

\section{Validation}

The questionnaire contains 18 questions covering three goals of the proposed solution. Goal 1 measures the benefit of architect mobility using SOA. Goal 2 checks the improvement in mobility in SOA by adding SU to the integrated architecture components (Make sure the effectiveness of SU). Goal 3 is designed to measure the risk of using untested mobile service that is recently created. The questions should be answered in likert scale i.e., very low, low, nominal, high and very high each effect (indicating 1, 2, 3, 4 and 5 respectively). The data analysis is based on sample size of thirty three (IT professionals and students).

Cumulative statistical analysis of Goal 1.

Table 3. Cumulative analysis of Goal 1.

\begin{tabular}{cccccc}
\hline Q. No. & Strong. disagree & Disagreed & Neutral & Agree & Strongly agree \\
\hline 1 & 12.50 & 6.25 & 31.25 & 15.63 & 34.38 \\
2 & 9.38 & 15.63 & 18.75 & 40.63 & 15.63 \\
3 & 9.38 & 9.38 & 28.13 & 40.63 & 12.50 \\
4 & 6.25 & 18.75 & 25.00 & 37.50 & 18.75 \\
5 & 9.38 & 12.50 & 21.88 & 37.50 & 12.50 \\
Total & 46.88 & 62.50 & 125.00 & 171.88 & 93.75 \\
Avg. & 9.38 & 12.50 & 25.00 & 34.38 & 18.75 \\
\hline
\end{tabular}

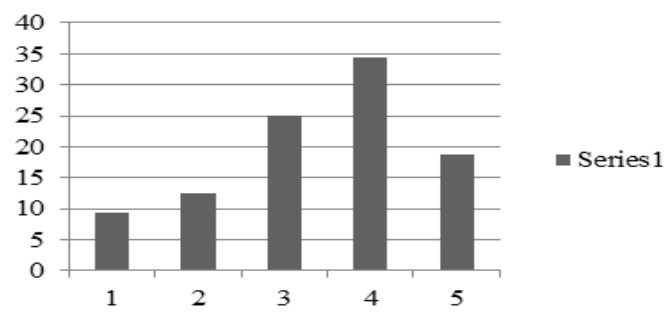

Fig.3. Cumulative Analysis of Goal 1. 
The questions in this goal measure how much SOA have capability to architect mobile application. Table 3 shows that $34.38 \%$ are high capability to architect mobile application whereas $18.75 \%$ are very high. Furthermore, $25 \%$ are neither high nor low. However, $12.5 \%$ are low while $9.38 \%$ are very low. Fig. 3 shows the results of Table 3 graphically.

\section{Cumulative statistical analysis of Goal 2.}

Table 4 gives information on questionnaire analysis table of goal 2. The questions in this goal check how much this paper improves SOA for mobility application by adding SU to the integrated architecture components and make sure the effectiveness of SU component. Almost 34\% see that SOA are high improved but not very high (16.02\%). Moreover, $31.64 \%$ are nominal improve. $11.33 \%$ and $7.42 \%$ as low and very low improve. Fig. 4 shows the results of Table 4 graphically.

Table 4. Cumulative Analysis for Goal 2.

\begin{tabular}{cccccc}
\hline Q. No & Strong. disagree & Disagreed & Neutral & Agree & Strongly agree \\
\hline 1 & 9.38 & 15.63 & 34.38 & 31.25 & 9.38 \\
2 & 3.13 & 3.13 & 31.25 & 43.75 & 18.75 \\
3 & 9.38 & 6.25 & 25.00 & 37.50 & 21.88 \\
4 & 21.88 & 9.38 & 34.38 & 25.00 & 9.38 \\
5 & 6.25 & 12.50 & 43.75 & 28.13 & 9.38 \\
6 & 6.25 & 12.50 & 40.63 & 28.13 & 12.50 \\
7 & 0.00 & 25.00 & 25.00 & 25.00 & 25.00 \\
8 & 3.13 & 6.25 & 18.75 & 50.00 & 21.88 \\
Total & 59.38 & 90.63 & 253.13 & 268.75 & 128.13 \\
Avg. & 7.42 & 11.33 & 31.64 & 33.59 & 16.02 \\
\hline
\end{tabular}

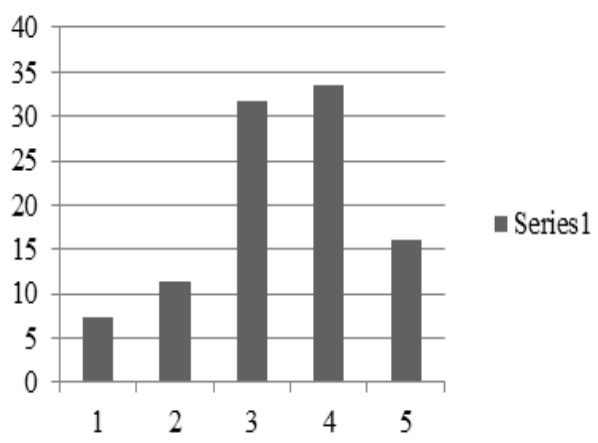

Fig.4. Cumulative Analysis of Goal 2.

\section{Cumulative statistical analysis of Goal 3.}

Table 5 shows $30.63 \%$ are high respondents' numbers of who will use untested new service for just the purpose of need rapid service creation whereas $13.75 \%$ are very high and $30 \%$ are nominal. $15.63 \%$ as the low percentage of respondents who is well using rapid untested service and $10 \%$ as very low. Fig. 5 shows this graphically as follows. 
Table 5. Cumulative Analysis of the Third Goal.

\begin{tabular}{cccccc}
\hline Q. No & Strong. disagree & Disagreed & Neutral & Agree & Strongly agree \\
\hline 1 & 12.50 & 28.13 & 31.25 & 18.75 & 9.38 \\
2 & 12.50 & 25.00 & 25.00 & 31.25 & 6.25 \\
3 & 9.38 & 9.38 & 34.38 & 31.25 & 15.63 \\
4 & 12.50 & 3.13 & 21.88 & 40.63 & 21.88 \\
5 & 3.13 & 12.50 & 37.50 & 31.25 & 15.63 \\
Total & 50.00 & 78.13 & 150.00 & 153.13 & 68.75 \\
Avg. & 10.00 & 15.63 & 30.00 & 30.63 & 13.75 \\
\hline
\end{tabular}

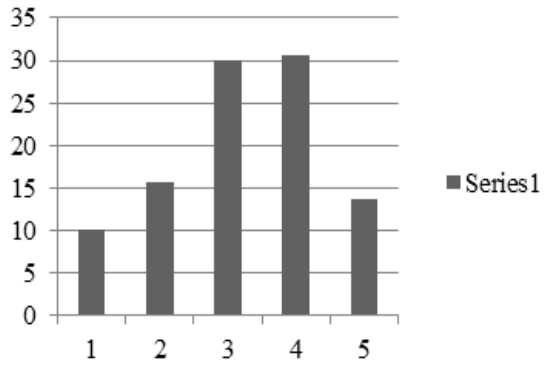

Fig.5. Cumulative Analysis of Goal 3.

Final Cumulative statistical analysis of 3 Goals.

Table 6 shows that $13.15 \%$ and 8.93 of the respondents as low and very low with this architecture and $28.88 \%$ of respondents are neither high nor low. As such, 49.04\% support is available. The results of Table 6 are displayed graphically in fig. 6.

Table 6. Final Cumulative Analysis of 3 Goals

\begin{tabular}{cccccc}
\hline $\begin{array}{c}\text { Likert Scale } \\
\text { Goals in } \% \\
\downarrow\end{array}$ & 1 & 2 & 3 & 4 & 5 \\
\hline Goal 1 & 9.38 & 12.50 & 25.00 & 34.38 & 18.75 \\
Goal 2 & 7.42 & 11.33 & 31.64 & 33.59 & 16.02 \\
Goal 3 & 10.00 & 15.63 & 30.00 & 30.63 & 13.75 \\
Total & 26.80 & 39.46 & 86.64 & 98.60 & 48.52 \\
Avg. & 8.93 & 13.15 & 28.88 & 32.87 & 16.17 \\
\hline
\end{tabular}

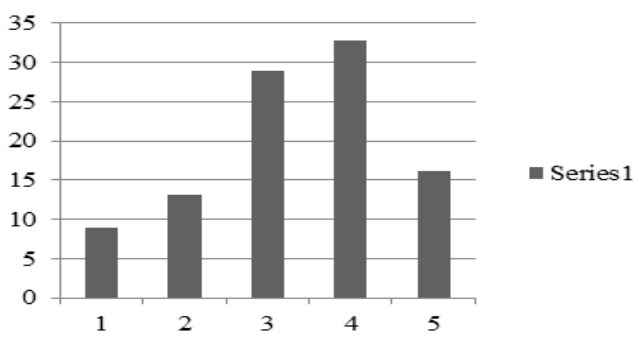

Fig.6. Final Cumulative Analysis of 3 Goals. 


\section{Conclusion}

SOA is suitable for mobile applications. Mobile Web Service Verifier component in SOA architecture has no action procedure when it does not find a best match of particular service in SP. However, it is lead to another point that mobile service creation speed in SOA architecture need to be increase. This paper integrated two enhancement SOA architectures and adding a new component to that architecture called SU as a solution for this restriction. It has many benefits such as give Mobile Web Service Verifier component action procedures in a situation of limited service in SP, raise the service creation speed and satisfy the consumer service needs. There are required for integrated architectures in order to SU component do it tasks such as SU needs Mobile Web Service Verifier component (it is included in one of the enhancements SOA architecture) to notify about a particular service that is not available to SP. As in validation, questionnaire used for validate the proposed SOA architecture. As such, $49.04 \%$ support is available to the proposed solution. It is a future work to implement the proposed component to test quantitatively by conducting a case study to check its effectiveness.

\section{References}

[1] A. Shrestha, "Mobile SOA Framework for Context-Aware Mobile Applications," in Mobile Data Management (MDM), 2010 Eleventh International Conference on, 2010, pp. 297-298.

[2] W. Romsaiyud and W. Premchaiswadi, "SOA context-aware mobile data model for emergency situation," in Knowledge Engineering, 2010 8th International Conference on ICT and, 2010, pp. 93-97.

[3] D. B. Hoang and C. Pham, "Connectivity abstractions and 'service-oriented network' architecture," in Computing, Networking and Communications (ICNC), 2012 International Conference on, 2012, pp. 337-342.

[4] J. Wang, "Exploiting mobility prediction for dependable service composition in wireless mobile ad hoc networks," Services Computing, IEEE Transactions on, vol. 4, no. 1, pp. 44-55, 2011.

[5] L. You, A. Belloni, G. Motta, and D. Sacco, "A System for Green Personal Integrated Mobility," in 2013 IEEE International Conference on Service Operations and Logistics, and Informatics, 2013, vol. 1.

[6] P. Choudhury, P. Dutta, S. Nandi, and N. C. Debnath, "Mobility aware distributed service composition framework in SOA based MANET application," in Industrial Informatics (INDIN), 2012 10th IEEE International Conference on, 2012, pp. 1016-1021.

[7] S. Abolfazli, Z. Sanaei, M. Shiraz, and A. Gani, "MOMCC: market-oriented architecture for mobile cloud computing based on service oriented architecture," in Communications in China Workshops (ICCC), 2012 1st IEEE International Conference on, 2012, pp. 8-13.

[8] W. D. Yu, A. Joshi, J. Patel, and V. Mehta, "A SOA Service Governance Approach to u-Healthcare System with Mobility Capability," in Communications (ICC), 2011 IEEE International Conference on, 2011, pp. 1-6.

[9] W. Rudametkin, L. Touseau, D. Donsez, and F. Exertier, "A framework for managing dynamic serviceoriented component architectures," in Services Computing Conference (APSCC), 2010 IEEE AsiaPacific, 2010, pp. 43-50.

[10] X. Hu, V. C. Leung, W. Du, B. Seet, and P. Nasiopoulos, "A Service-oriented Mobile Social Networking Platform for Disaster Situations," in System Sciences (HICSS), 2013 46th Hawaii International Conference on, 2013, pp. 136-145.

[11] Y. Liu, C. Xu, and S. Cheung, "Diagnosing Energy Efficiency and Performance for Mobile Internetware Applications: Challenges and Opportunities."

[12] N. Ali, F. Chen, and C. Solis, "Modeling Support for Mobile Ambients in Service Oriented Architecture," in Mobile Services (MS), 2012 IEEE First International Conference on, 2012, pp. 1-8.

[13] M. A. Serhani and A. Benharref, "MSOA: Mobility-aware Service Oriented Architecture," in Services Computing Conference (APSCC), 2011 IEEE Asia-Pacific, 2011, pp. 345-352. 
[14] M. Sherriff, "Teaching web services and service-oriented architecture using mobile platforms," in Frontiers in Education Conference (FIE), 2010 IEEE, 2010, p. S2D-1.

[15] N. B. Mabrouk, N. Georgantas, and V. Issarny, "A semantic end-to-end QoS model for dynamic service oriented environments," in Proceedings of the 2009 ICSE Workshop on Principles of Engineering Service Oriented Systems, 2009, pp. 34-41.

[16] Shuai, Qin; Zhou Ming-Quan., "Cloud computing promotes the progress of m-learning," Uncertainty Reasoning and Knowledge Engineering (URKE), International Conference on IEEE, vol.2, no., pp.162164, 4-7 Aug. 2011.

[17] Hirsch, Benjamin; Jason WP Ng., "Education beyond the Cloud: Anytime-anywhere learning in a smart campus environment," Internet Technology and Secured Transactions (ICITST), International Conference on IEEE, vol., no., pp.718-723, 11-14 Dec. 2011.

[18] M. Tantow: Cloud Computing and Smartphones. Cloud Times. http://cloudtimes.org/2011/03/01/cloudcomputing-and-smartphones/ (01 March 2011). Accessed 2014.

[19] Chen, Shaoyong; Min Lin; Huanming Zhang, "Research of mobile learning system based on cloud computing," e-Education, Entertainment and e-Management (ICEEE), International Conference on IEEE, vol., no., pp.121-123, 27-29 Dec. 2011.

[20] Ferzli, Rony; Ibrahim Khalife;, "Mobile cloud computing educational tool for image/video processing algorithms," Digital Signal Processing Workshop and IEEE Signal Processing Education Workshop (DSP/SPE), IEEE, vol., no., pp.529- 533,4-7 Jan. 2011.

[21] Veerabhadram, Paduri; Pieter Conradie, "Mobile cloud framework architecture for education institutions," Science and Information Conference (SAI), IEEE, vol., no., pp.924-927, 7-9 Oct. 2013.

[22] Lee, Jae Dong; Jong-Hyuk Park; , "Application for Mobile Cloud Learning," Network-Based Information Systems (NBIS), In proc. 16th IEEE Int. Conf., pp. 296-299, 4-6 Sept. 2013.

\section{Authors' Profiles}

Dalal F. Aljebry is a student of master in IT from the Department of Information Technology, Faculty of Computing and Information Technology, King Abdulaziz University, Jeddah, Saudi Arabia.

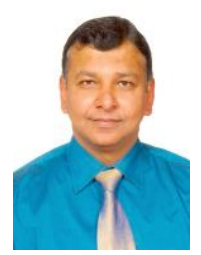

Dr. Rizwan J. Qureshi received his Ph.D. degree from National College of Business Administration \& Economics, Pakistan 2009. He is currently working as an Associate Professor in the Department of IT, King Abdulaziz University, Jeddah, Saudi Arabia. This author is the best researcher awardees from the Department of Information Technology, King Abdulaziz University in 2013 and 2016. He is also honoured as the best researcher from the Department of Computer Science, COMSATS Institute of Information Technology, Pakistan in 2008.

How to cite this paper: Dalal Aljebry, Rizwan Qureshi,"Proposal to Improve Mobility in Service Oriented Architecture", International Journal of Education and Management Engineering(IJEME), Vol.7, No.1, pp.14-24, 2017.DOI: 10.5815/ijeme.2017.01.02 